\title{
SNAPSHOT How do you like your coffee?
}

This floating fractal (top, left) is formed 90 seconds after a drop of instant coffee falls into a cup of milk.

Coffee is heavier than milk and the battle between gravity and surface tension plays out at the boundary between the two liquids. The coffee falls vertically through the milk (bottom, left, with water replacing milk for ease of viewing), and the fractal pattern emerges.

The pattern constantly shifts as parts of it are sucked into the milk, producing a fractal structure with the same dimension as a Sierpiński carpet - formed when a square is cut into nine identical squares; the central square is removed; and the procedure is repeated with the remaining eight squares and so on infinitely.

Michiko Shimokawa and Shonosuke Ohta, fluid scientists at Kyushu University in Fukuoka City, Japan, say that it is the first time this kind of fractal has been shown experimentally (www.arxiv.org/abs/0809.2458), and they managed to recreate the process using a magnetic liquid instead of coffee (far right).

Katharine Sanderson
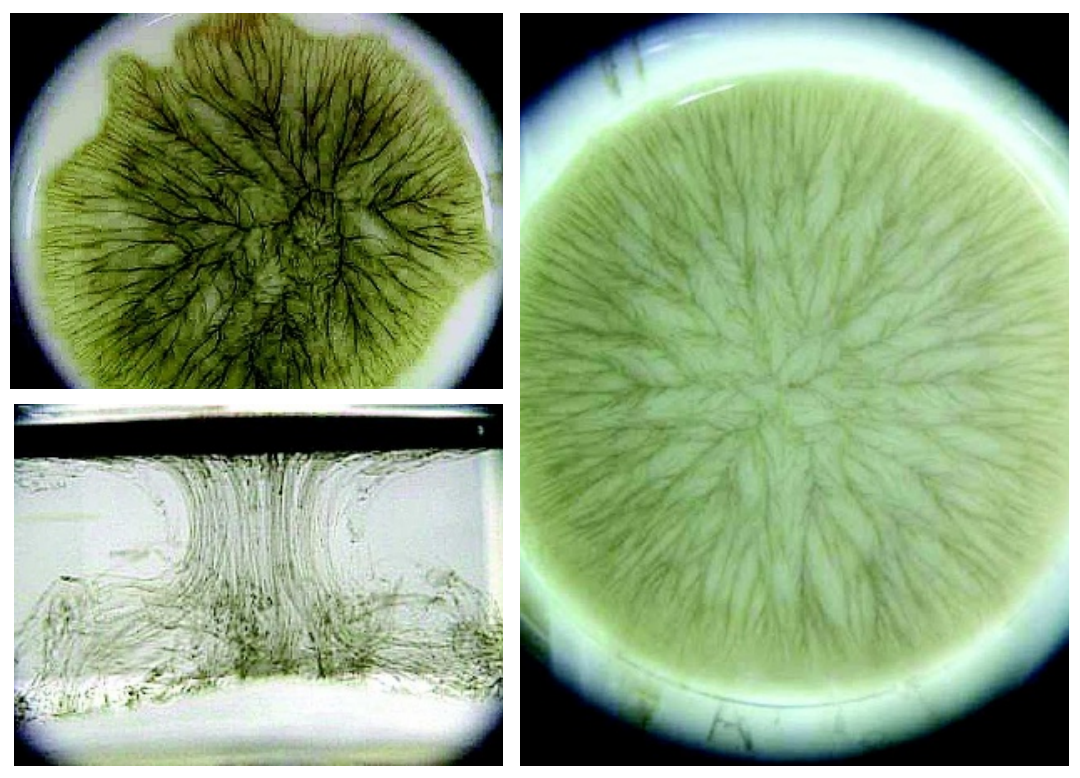

ATOM REFLECTIONS

Ultrasmooth mirror could

herald birth of a new

microscope.

www.nature.com/news

Katharine Sanderson 